PHYSICAL REVIEW D 89, 045001 (2014)

\title{
Dynamical sectors of a relativistic two particle model
}

\author{
Daniele Dominici* \\ Department of Physics and Astronomy, University of Florence, 50019 Sesto Fiorentino, Firenze, Italy \\ and Sezione di Firenze, INFN, 50019 Sesto Fiorentino, Firenze, Italy \\ Joaquim Gomis ${ }^{\dagger}$ \\ Theory Group, Physics Department, CERN, CH1211, Geneva 23, Switzerland \\ and Departament d'Estructura i Constituents de la Matèria and Institut de Ciències del Cosmos, \\ Universitat de Barcelona, Diagonal 645, 08028 Barcelona, Spain \\ Kiyoshi Kamimura \\ Department of Physics, Toho University, Miyama, Funabashi 274-8510, Japan \\ Giorgio Longhi ${ }^{\S}$ \\ Department of Physics and Astronomy, University of Florence, 50019 Sesto Fiorentino, Firenze, Italy \\ and Sezione di Firenze, INFN, 50019 Sesto Fiorentino, Firenze, Italy \\ (Received 16 December 2013; published 3 February 2014)

\begin{abstract}
We reconsider a model of two relativistic particles interacting via a multiplicative potential, as an example of a simple dynamical system with sectors, or branches, with different dynamics and degrees of freedom. The presence or absence of sectors depends on the values of rest masses. Some aspects of the canonical quantization are described. The model could be interpreted as a bigravity model in one dimension.
\end{abstract}

DOI: 10.1103/PhysRevD.89.045001

PACS numbers: 11.10.Ef, 11.30.Cp, 12.39.Pn

\section{INTRODUCTION}

Usually a physical system has a definite number of physical degrees of freedom. However it sometimes happens that it has sectors, i.e., branches of phase spaces that have different canonical structures, such as different physical degrees of freedom, or gauge symmetries. Generically, these sectors appear for dynamical systems when the rank of the matrix of Poisson brackets of primary constraints is not constant, in other words when the regularity conditions are not verified; see for example [1]. In these cases there are nonunique solutions of the stabilization condition in the Dirac's algorithm of constraints. The presence of sectors was also analyzed in other contexts, for example, when the Legendre transformation and the Hamiltonian are multivalued [2-5].

A model of the Zwei-Dreibein gravity has been proposed in a very recent paper [6]. The presence or absence of the Boulware-Deser mode in the model has been reanalyzed in [7]; the Hamiltonian analysis shows in general the existence of sectors, and, in each sector, the system has different number of degrees of freedom. For a suitable choice of the parameters there appears only one sector where the Boulware-Deser mode is absent. ${ }^{1}$ In the case of four dimensions, bigravity contains

\footnotetext{
*dominci@fi.infn.it

†gomis@ecm.ub.es

kamimura@ph.sci.toho-u.ac.jp

\$longhi@fi.infn.it
}

also sectors; see for example [8]. For other models with sectors see [9-12].

In order to have a better understanding of how to deal with dynamical systems with sectors at classical and quantum levels, we consider a simple model of two relativistic particles [13-16]. The model is described by a sum of two relativistic particle Lagrangians in which the interaction is introduced by replacing their rest masses with potentials that depend on the Minkowskian distance of the two particles, ${ }^{2}$

$$
\begin{aligned}
L & =-\sqrt{\left(m_{10}^{2}-V\left(r^{2}\right)\right) \dot{x}_{1}^{2}}-\sqrt{\left(m_{20}^{2}-V\left(r^{2}\right)\right) \dot{x}_{2}^{2}} \\
& =-\sum_{j=1,2} \sqrt{m_{j}^{2}\left(r^{2}\right) \dot{x}_{j}^{2}},
\end{aligned}
$$

where $x_{j}(\tau),(j=1,2)$ are the space-time coordinates of the two particles. $V\left(r^{2}\right)$ is any Poincaré invariant function of the squared relative distance $r^{2}=\left(x_{2}-x_{1}\right)^{2} \cdot m_{j 0}$ 's are the rest masses of the particles and $m_{j}^{2}\left(r^{2}\right)=m_{j 0}^{2}-V\left(r^{2}\right)$ are the effective masses of the particles. The interaction breaks the individual invariance under diffeomorphism (Diff) of the action of two free particles, leaving a universal Diff invariance.

\footnotetext{
${ }^{1}$ We acknowledge Eric Bergshoeff and Paul Townsend for discussions on this point.

${ }^{2}$ Here the metric is $(+;---)$.
} 
The canonical action is given by

$$
\begin{aligned}
S & =\int d \tau \sum_{j=1,2}\left(p_{j} \dot{x}_{j}-\frac{e_{j}}{2}\left(p_{j}^{2}-m_{j}^{2}\left(r^{2}\right)\right)\right) \\
& =\int d \tau \sum_{j=1,2}\left(p_{j} \dot{x}_{j}-e_{j} \varphi_{j}\right),
\end{aligned}
$$

where $e_{j}$ are Lagrangian multipliers that we can interpret as two einbein variables, and $\phi_{j}=1 / 2\left(p_{j}^{2}-m_{j}^{2}\left(r^{2}\right)\right)$ are the mass-shell constraints.

The matrix of the Poisson brackets of the primary constraints reads

$$
\left(\begin{array}{ll}
\left\{\varphi_{1}, \varphi_{1}\right\} & \left\{\varphi_{1}, \varphi_{2}\right\} \\
\left\{\varphi_{2}, \varphi_{1}\right\} & \left\{\varphi_{2}, \varphi_{2}\right\}
\end{array}\right)=\left(\begin{array}{cc}
0 & -V^{\prime}\left(r^{2}\right)(P \cdot r) \\
V^{\prime}\left(r^{2}\right)(P \cdot r) & 0
\end{array}\right),
$$

where $P=p_{1}+p_{2}$ is the total momenta of the system and $V^{\prime}\left(r^{2}\right)=\frac{d}{d r^{2}} V\left(r^{2}\right)$ and has not constant rank. The rank is 2 if $V^{\prime}\left(r^{2}\right)(P \cdot r) \neq 0$ and it is 0 if $V^{\prime}\left(r^{2}\right)(P \cdot r)=0$; therefore, the model has sectors. ${ }^{3}$ The detailed structure of the sectors will be studied in the next section for the case of the harmonic oscillator.

If we eliminate the momenta $p_{i}$ by using their equations of motion we get

$$
L=\sum_{j=1,2}\left(\frac{\dot{x}_{j}^{2}}{2 e_{j}}+\frac{e_{j}}{2} m_{j}^{2}\left(r^{2}\right)\right) .
$$

In this form the Lagrangian can be reinterpreted as the Lagrangian of a model of bigravity in one dimension. Note that for this Lagrangian the primary constraints $\phi_{i}$ of (1) appear as secondary constraints. If we restrict the $V\left(r^{2}\right)$ to a harmonic potential, it can be shown that, in the case of equal rest masses $\left(m_{10}=m_{20} \equiv m_{0}\right)$, the model has a new extra Noether gauge symmetry, in addition to Diff. The existence of two gauge symmetries depending on the phase space regions, $P^{2}=0$ or $P^{2}>0$, is a consequence of the fact that the model has sectors, with different degrees of freedom in each sector.

In this paper we study in detail the appearance of sectors at the Hamiltonian level. As we will see for unequal rest masses there is only one sector. It is a massive sector in the sense that $P^{2} \neq 0$. This sector contains first and second class constraints. The physical degrees of freedom are those of a system of two massive particles. If the rest masses are equal, we have three sectors: one with $P^{2} \neq 0$ like in the previous case and two sectors with $P^{2}=0$ with different numbers of first and second class constraints. Although the massless sectors are empty (no classical solution) for

\footnotetext{
${ }^{3}$ The presence of sectors was already noticed in [13-16] but only one sector was studied in detail.
}

positive rest mass square $\left(m_{0}^{2}>0\right)$, they are not so for tachyonic rest masses $\left(m_{0}^{2}<0\right)$.

We perform the canonical quantization of the two sectors. For the massive sector it is useful to consider first a canonical transformation at the classical level $[14,16]$ such that the second class constraints become a pair of canonical variables $[17,18]$. This allows one to impose the second class constraints on the physical states, by considering a non-Hermitean combination of them. We get a spectrum of increasing masses for higher internal spins. In the case of unequal rest masses there is a branch of the mass spectrum that contains ghosts. Instead, for the massless sector, there are no physical states for physical particles with a positive rest mass square corresponding to the fact that there are no classical solutions. If we consider tachyons this sector is not empty; there are states with helicities depending on the value of the tachyonic mass.

The organization of this paper is as follows. In Sec. 2 we introduce the model and perform the Hamiltonian analysis. In Sec. 3 we give the gauge transformations. Section 4 is devoted to the canonical quantization and finally in Sec. 5 we give some conclusions and an outlook.

\section{SECTORS OF AN INTERACTING RELATIVISTIC TWO PARTICLE MODEL}

We reconsider a model of two relativistic interacting particles via a multiplicative potential introduced in [13-16]. The Lagrangian is given in (1). We rewrite the Lagrangian by introducing two einbein variables $e_{j}$ (see for example $[19,20])$

$$
L=\sum_{j=1,2}\left(\frac{\dot{x}_{j}^{2}}{2 e_{j}}+\frac{e_{j}}{2} m_{j}^{2}\left(r^{2}\right)\right) .
$$

In order to have a well-defined Lagrangian we will assume $e_{j} \neq 0$. In addition $e_{1}, e_{2}$ should have the same sign in order to reproduce (1), so $e_{1}+e_{2} \neq 0$. In this form the model can be reinterpreted as a bigravity in one dimension. In this paper we will consider a special case of harmonic potential $m_{j}^{2}\left(r^{2}\right)=m_{j 0}^{2}-\kappa^{2} r^{2}$ with the parameters, the rest masses $m_{j 0}^{2}$ and $\kappa$. The Lagrange equations of motion are

$$
\begin{aligned}
{[L]_{x_{j}} } & =\frac{\partial L}{\partial x_{j}}-\frac{d}{d \tau} \frac{\partial L}{\partial \dot{x}_{j}} \\
& =(-)^{j+1} \kappa^{2}\left(e_{1}+e_{2}\right) r+\frac{\dot{e}_{j} \dot{x}_{j}}{e_{j}^{2}}-\frac{\ddot{x}_{j}}{e_{j}}=0, \\
{[L]_{e_{j}} } & =\frac{\partial L}{\partial e_{j}}-\frac{d}{d \tau} \frac{\partial L}{\partial \dot{e}_{j}}=\frac{1}{2}\left(\frac{\dot{x}_{j}^{2}}{e_{j}^{2}}-m_{j}^{2}\right)=0 .
\end{aligned}
$$

In the Hamiltonian formalism the canonical momenta are

$$
p_{j}=\frac{\partial L}{\partial \dot{x}_{j}}=\frac{\dot{x}_{j}}{e_{j}}, \quad \pi_{j}=\frac{\partial L}{\partial \dot{e}_{j}}=0
$$


and we have two primary constraints

$$
\pi_{j}=0, \quad(j=1,2) .
$$

The canonical Hamiltonian is given by

$$
H_{c}=e_{j} \phi_{j}, \quad \phi_{j} \equiv \frac{1}{2}\left(p_{j}^{2}-m_{j}^{2}\left(r^{2}\right)\right),
$$

and the Dirac Hamiltonian, that includes the primary constraints, is

$$
H=H_{c}+\pi_{j} \Lambda_{j}
$$

where $\Lambda_{j}$ 's are arbitrary functions of $\tau$ that work as Lagrangian multipliers. The Hamilton equations of motion are

$$
\begin{aligned}
& \dot{e}_{j}=\Lambda_{j}, \quad \dot{x}_{j}=e_{j} p_{j}, \quad \dot{\pi}_{j}=-\phi_{j}, \\
& \dot{p}_{j}=(-1)^{j+1}\left(e_{1}+e_{2}\right) \kappa^{2} r,
\end{aligned}
$$

which imply that the total momentum $P=p_{1}+p_{2}$ is conserved.

The Dirac's algorithm starts to examine the stability condition of the primary constraints $\pi_{j}=0$. It produces the secondary constraints

$$
\phi_{j}=0
$$

and the stability of these constraints gives

$$
\dot{\phi}_{1}=\kappa^{2}(P \cdot r) e_{2}=0, \quad \dot{\phi}_{2}=-\kappa^{2}(P \cdot r) e_{1}=0 .
$$

Note that the matrix Poisson brackets of the secondary constraints has no constant rank, in fact $\left\{\phi_{1}, \phi_{2}\right\}=$ $\kappa^{2}(P \cdot r)$.

Since $e_{j}$ 's are not vanishing by initial hypothesis of the Lagrangian (5), in addition $e_{1}=e_{2}=0$ only gives trivial system $H \equiv 0$, we have a tertiary constraint

$$
\Theta \equiv(P \cdot r)=0
$$

Now we compute the Poisson bracket of $\Theta$ with $\phi_{i}$

$$
\begin{aligned}
& \left(\left\{\Theta, \phi_{1}\right\},\left\{\Theta, \phi_{2}\right\}\right) \\
& =\left(-\frac{1}{2} P^{2}-\frac{1}{2}\left(m_{10}^{2}-m_{20}^{2}\right),-\frac{1}{2} P^{2}+\frac{1}{2}\left(m_{10}^{2}-m_{20}^{2}\right)\right),
\end{aligned}
$$

where the constraints $\phi_{i}=0$ have been used. The above rectangular matrix does not have a constants rank. It has rank 0 for $P^{2}=m_{10}^{2}-m_{20}^{2}=0$ and rank 1 for others; therefore, the model has further ramifications.

The stability of the tertiary constraint requires the relation

$$
\begin{aligned}
\chi= & \dot{\Theta}=P \cdot\left(e_{2} p_{2}-e_{1} p_{1}\right) \\
= & -\frac{\left(e_{1}-e_{2}\right)}{2} P^{2} \\
& -\left(e_{1}+e_{2}\right)\left(\phi_{1}-\phi_{2}+\frac{1}{2}\left(m_{1}^{2}\left(r^{2}\right)-m_{2}^{2}\left(r^{2}\right)\right)\right) \\
= & -\frac{1}{2}\left(e_{-} P^{2}+e_{+} \Delta_{-}\right)=0,
\end{aligned}
$$

where we have used the secondary constraints $\phi_{j}=0$ and defined

$$
e_{ \pm}=e_{1} \pm e_{2}, \quad \Delta_{-}=\left(m_{10}^{2}-m_{20}^{2}\right)
$$

Now we study the different sectors of this constraint depending on the values of $\Delta_{-}$. For the model with different rest masses $\Delta_{-} \neq 0$, since $e_{+} \neq 0$, (17) implies $P^{2} \neq 0$. Solving it for $e_{-}$, the quaternary constraint takes the form

$$
e_{-}+\frac{\Delta_{-}}{P^{2}} e_{+}=0 \quad \text { (massive sector) }
$$

The stability of this constraint gives a relation between the arbitrary functions,

$$
\Lambda_{-}+\frac{\Delta_{-}}{P^{2}} \Lambda_{+}=0
$$

There is only one sector for $\Delta_{-}=\left(m_{10}^{2}-m_{20}^{2}\right) \neq 0$. The first class combinations of constraints are

$$
\pi_{+}-\frac{\Delta_{-}}{P^{2}} \pi_{-}=0, \quad \phi_{+}-\frac{\Delta_{-}}{P^{2}} \phi_{-}=0,
$$

with $\phi_{ \pm}=\phi_{1} \pm \phi_{2}$, and

$$
\begin{aligned}
& \pi_{-}=0, \quad e_{-}+\frac{\Delta_{-}}{P^{2}} e_{+}=0, \\
& \phi_{-}=-(P \cdot q)-\frac{\Delta_{-}}{2}=0, \quad \Theta=(P \cdot r)=0
\end{aligned}
$$

are the second class constraints, where $q=\frac{1}{2}\left(p_{2}-p_{1}\right)$ is the relative momentum.

For the case of equal rest masses $\Delta_{-}=$ $\left(m_{10}^{2}-m_{20}^{2}\right)=0,(17)$ requires either

$$
\begin{aligned}
& \text { case 1: } \quad e_{-}=0, \quad P^{2} \neq 0, \quad \text { then } \Lambda_{-}=0 \quad \text { (massive sector), } \\
& \text { case 2: } \quad P^{2}=0, \quad e_{-} \neq 0 \quad \text { (no more cond.: massless sector), }
\end{aligned}
$$

case 3: $P^{2}=0$ and $e_{-}=0, \quad$ then $\Lambda_{-}=0 \quad$ (massless sector). 
Case 1 is just $\Delta_{-}=0$ of the previous case (19). The constraints appearing here

$$
\pi_{+}=0, \quad \phi_{+}=0
$$

are first class while

$\pi_{-}=0, \quad e_{-}=0, \quad \phi_{-}=-(P \cdot q)=0, \quad(P \cdot r)=0$

are second class. The constraint $\phi_{+}=0$ determines the mass of the system, while the last two kill the longitudinal relative coordinate $r^{\mu}$ and momentum $q^{\mu}$. The physical degrees of freedom are given by

$$
2 \times 2+8 \times 2-2 \times 2-4=12,
$$

which is the physical dimension of a system of two massive particles.

In case 2 all the constraints

$$
\pi_{ \pm}=0, \quad \phi_{ \pm}=0, \quad(P \cdot r)=0, \quad P^{2}=0
$$

are first class and the dimension of the physical phase space is

$$
2 \times 2+8 \times 2-2 \times 6=8,
$$

which is the physical phase space of a system with two massless particles.

In the case 3 the constraints

$$
\pi_{+}=0, \quad \phi_{ \pm}=0, \quad(P \cdot r)=0, \quad P^{2}=0
$$

are first class and

$$
\pi_{-}=0, \quad e_{-}=0
$$

are second class. In this case the dimension of the physical phase space is

$$
2 \times 2+8 \times 2-2 \times 5-2=8
$$

which is the same as in the (above) case 2. Despite the fact that counting of degrees of freedom is eight, as we will see in the next section, the constraints have no solution for positive rest mass case, $m_{0}^{2}>0$, thus these sectors are empty.

Now we would like to see which is the evolution of the Lagrangian multipliers in the different sectors. If $e_{-}=0$ at $\tau=0$, integrating (12) the evolution in $\tau$ is given by

$$
e_{-}=\int_{0}^{\tau} \Lambda_{-}\left(\tau^{\prime}\right) d \tau
$$

which, for generic $\Lambda_{-}$, gives $e_{-} \neq 0$. However since in the case 1 and $3 \Lambda_{-}=0, e_{-}$will remain zero during the evolution of the system. Instead, if we are in case $2, P^{2}=0$, $e_{-} \neq 0$. If $e_{-}(0)=a$ at $\tau=0$, the evolution in this case is given by

$$
e_{-}=\int_{0}^{\tau} \Lambda_{-}\left(\tau^{\prime}\right) d \tau^{\prime}+a
$$

We always can choose $\Lambda_{-}$such that at given $\tau$ we have $e_{-}(\tau)=0$ and we are no longer in case 2 . However we are not in case 3 , since in this case too $\Lambda_{-}=0$. The system will evolve and it could move again to $e_{-} \neq 0$. It seems that from the point of view of the true degrees of freedom the evolution among sectors does not matter since the spacetime variables are unaffected by this phenomenon. Evolution among sectors occurs in the model studied in $[11] .^{4}$

\section{GAUGE SYMMETRIES}

Let us now study the gauge symmetries in these sectors. In the massive sector with equal or different rest masses we have only one gauge transformation in agreement with the presence of only one primary first class constraint. The transformation is given by

$$
\delta e_{j}=\frac{d}{d \tau}\left(\epsilon e_{j}\right), \quad \delta x_{j}=\left(\epsilon e_{j}\right) p_{j}=\epsilon \dot{x}_{j},
$$

where $\epsilon=\epsilon(\tau)$ is an arbitrary function. It is a Noether symmetry since

$$
\begin{aligned}
\delta L & =\frac{\dot{x}_{j}}{e_{j}} \frac{d}{d \tau}\left(\epsilon \dot{x}_{j}\right)-\frac{\dot{x}_{j}^{2}}{2 e_{j}^{2}} \frac{d}{d \tau}\left(\epsilon e_{j}\right)+\frac{m^{2}}{2} \frac{d}{d \tau}\left(\epsilon e_{j}\right)-e_{j} \kappa^{2} r(\epsilon \dot{r}) \\
& =\frac{d}{d \tau}(\epsilon L) .
\end{aligned}
$$

It is the well-known diffeomorphism transformation that has a closed algebra

$$
\begin{aligned}
& {\left[\delta_{\epsilon_{2}}, \delta_{\epsilon_{1}}\right] e_{j}=\delta_{\epsilon_{3}} e_{j}, \quad\left[\delta_{\epsilon_{2}}, \delta_{\epsilon_{1}}\right] x_{j}=\delta_{\epsilon_{3}} x_{j},} \\
& \epsilon_{3}=\epsilon_{1} \dot{\epsilon}_{2}-\epsilon_{2} \dot{\epsilon}_{1} .
\end{aligned}
$$

For the massless sector (24), with equal masses, we have two independent primary first class constraints. This is a signal that we will have two gauge transformations, one being the Diff as in the massive case (36) and the other one a new transformation with gauge parameter $\lambda(\tau)$,

\footnotetext{
${ }^{4}$ We acknowledge discussions with Jorge Zanelli on this point.
} 


$$
\begin{aligned}
& \delta e_{j}=(-)^{j+1}\left[\frac{d}{d \tau}\left\{\frac{1}{\kappa^{2}\left(e_{1}+e_{2}\right)} \frac{d}{d \tau}\left(\frac{-1}{\left(e_{1}-e_{2}\right)} \dot{\lambda}\right)\right\}-\frac{\left(e_{1}+e_{2}\right)}{\left(e_{1}-e_{2}\right)} \dot{\lambda}\right], \\
& \delta x_{j}=(-)^{j+1}\left\{\frac{1}{\kappa^{2}\left(e_{1}+e_{2}\right)} \frac{d}{d \tau}\left(\frac{-1}{\left(e_{1}-e_{2}\right)} \dot{\lambda}\right)\right\} \frac{\dot{x}_{j}}{e_{j}}+\frac{1}{\left(e_{1}-e_{2}\right)} \dot{\lambda} r+\lambda\left(\frac{\dot{x}_{1}}{e_{1}}+\frac{\dot{x}_{2}}{e_{2}}\right),
\end{aligned}
$$

which is also a Noether symmetry, indeed,

$$
\delta L=\frac{d}{d \tau}\left[\frac{1}{2}\left\{\frac{1}{\kappa^{2}\left(e_{1}+e_{2}\right)} \frac{d}{d \tau}\left(\frac{-1}{\left(e_{1}-e_{2}\right)} \dot{\lambda}\right)\right\}\left\{\left(\frac{\dot{x}_{1}}{e_{1}}\right)^{2}-\left(\frac{\dot{x}_{2}}{e_{2}}\right)^{2}\right\}+\frac{\lambda}{2}\left(\frac{\dot{x}_{1}}{e_{1}}+\frac{\dot{x}_{2}}{e_{2}}\right)^{2}\right] .
$$

In this case the algebra of gauge transformation closes only on shell as

$$
\left[\delta_{\lambda_{2}}, \delta_{\lambda_{1}}\right] e_{i}=\delta_{\lambda_{3}} e_{i}, \quad\left[\delta_{\lambda_{2}}, \delta_{\lambda_{1}}\right] x_{i}=\delta_{\lambda_{3}} x_{i}-\frac{\epsilon_{i j}[L]_{x_{j}}}{\kappa^{2} e_{1} e_{2}} \frac{d}{d \tau}\left(\frac{\left(\lambda_{1} \dot{\lambda}_{2}-\lambda_{2} \dot{\lambda}_{1}\right)}{\left(e_{1}-e_{2}\right)}\right)
$$

where $[L]_{x}$ are the equations of motion for $x_{j}(6)$ and

$$
\lambda_{3}=\frac{2}{\kappa^{2}\left(e_{1}+e_{2}\right)\left(e_{1}-e_{2}\right)^{2}}\left(\dot{\lambda}_{1} \ddot{\lambda}_{2}-\dot{\lambda}_{2} \ddot{\lambda}_{1}\right)
$$

The second gauge transformation $\left(\delta_{\lambda}\right)$ and diffeomorphism transformation $\left(\delta_{\epsilon}\right)$ close off shell as

$$
\left[\delta_{\epsilon}, \delta_{\lambda}\right] e_{j}=\delta_{\tilde{\lambda}} e_{j}, \quad\left[\delta_{\epsilon}, \delta_{\lambda}\right] x_{j}=\delta_{\tilde{\lambda}} x_{j}, \quad \tilde{\lambda}=-\epsilon \dot{\lambda}
$$

Note that the second gauge transformation exists only when $e_{ \pm}$are not vanishing. The gauge structure of a theory is encoded in the BV formalism [21]. For a review, see for example $[22,23]$. The construction of the classical master equation and the BRST symmetry will be given elsewhere [24].

\section{CANONICAL QUANTIZATION}

Here we will discuss the canonical quantization of the model for the massive and massless sectors.

\section{A. Massive sector}

The quantization of the massive sector of the model for unequal rest masses $\left(\Delta_{-} \neq 0\right)$, and also for equal mass case $\left(\Delta_{-}=0\right)$, is performed following [16]. The constraints in (21)-(22) are

$\pi_{+}-\frac{\Delta_{-}}{P^{2}} \pi_{-}=0, \quad \phi_{+}-\frac{\Delta_{-}}{P^{2}} \phi_{-}=0$

$$
\begin{gathered}
\pi_{-}=0, \quad e_{-}+\frac{\Delta_{-}}{P^{2}} e_{+}=0, \\
\phi_{-}=-(P \cdot q)-\frac{\Delta_{-}}{2}=0, \\
\Theta=(P \cdot r)=0, \quad(2 \mathrm{cl} .) .
\end{gathered}
$$

Since the latter four are the second class constraints, we first perform a canonical transformation such that they become new sets of canonical pairs. ${ }^{5}$ It is generated by

$$
\begin{aligned}
W(\pi, P, q, \bar{e}, \bar{x}, u)= & \frac{1}{2} \bar{e}_{+}\left(\pi_{+}-\frac{\Delta_{-}}{P^{2}} \pi_{-}\right)+\frac{1}{2} \bar{e}_{-} \pi_{-} \\
& +\bar{x}^{\mu} P_{\mu}+u^{(\lambda)} \epsilon_{(\lambda)}{ }^{\mu}(P) q_{\mu} \\
& +u^{(0)} \frac{\Delta_{-}}{2 \sqrt{P^{2}}},
\end{aligned}
$$

where the polarization vectors $\epsilon_{(\lambda)}{ }^{\mu},(\lambda=0,1,2,3$ are

$\epsilon_{(\lambda)}{ }^{\mu}=\left(\begin{array}{c}\epsilon_{(0)}{ }^{\mu} \\ \epsilon_{\left(\lambda^{\prime}\right)}{ }^{\mu}\end{array}\right)=\left(\begin{array}{cc}\frac{P^{0}}{\sqrt{P^{2}}} & \frac{P^{j}}{\sqrt{P^{2}}} \\ \frac{P_{\lambda^{\prime}}}{\sqrt{P^{2}}} & \delta_{\lambda^{\prime}}{ }^{j}-\frac{P_{\lambda^{\prime}} P^{j}}{\sqrt{P^{2}}\left(P_{0}+\sqrt{P^{2}}\right)}\end{array}\right)$,

and $\lambda^{\prime}=1,2,3, j=1,2,3$. The new canonical variables $\left(\bar{e}_{i}, \bar{\pi}_{i}, \bar{x}^{\mu}, \bar{P}_{\mu}, u^{(\lambda)}, v_{(\lambda)}\right)$ are related to the old ones $\left(e_{i}, \pi_{i}, x^{\mu}=\frac{1}{2}\left(x_{1}^{\mu}+x_{2}^{\mu}\right), P_{\mu}, r^{\mu}, q_{\mu}\right)$ by [16]

\footnotetext{
${ }^{5}$ The theorem that guarantees the existence of this canonical transformation is given by $[17,18]$.
} 


$$
\begin{aligned}
\bar{\pi}_{+} & =\frac{\partial W}{\partial \bar{e}_{+}}=\left(\pi_{+}-\frac{\Delta_{-}}{P^{2}} \pi_{-}\right), \quad \bar{\pi}_{-}=\frac{\partial W}{\partial \bar{e}_{-}}=\pi_{-}, \\
e_{+} & =\bar{e}_{+}, \quad e_{-}=\bar{e}_{-}-\bar{e}_{+} \frac{\Delta_{-}}{P^{2}} \\
x^{\mu} & =\frac{\partial W}{\partial P_{\mu}} \\
& =\bar{x}^{\mu}+u^{(\lambda)} \frac{\partial \epsilon_{(\lambda)}{ }^{\nu}}{\partial P_{\mu}} q_{\nu}-\frac{\Delta_{-} u^{(0)}}{2 \sqrt{P^{2}}} P^{\mu}+\bar{e}_{+} \frac{2 \Delta_{-}}{\left(P^{2}\right)^{2}} \pi_{-} P_{\mu}, \\
\bar{P}_{\mu} & =\frac{\partial W}{\partial \bar{x}^{\mu}}=P_{\mu}, \quad r^{\mu}=\frac{\partial W}{\partial q_{\mu}}=u^{(\lambda)} \epsilon_{(\lambda)}{ }^{\mu} \\
v_{(\lambda)} & =\frac{\partial W}{\partial u^{(\lambda)}}=\epsilon_{(\lambda)}{ }^{\mu} q_{\mu}+\frac{\Delta_{-}}{2 \sqrt{P^{2}}} \delta_{\lambda}{ }^{0} .
\end{aligned}
$$

The second class constraints in (45) are

$$
\begin{aligned}
\bar{\pi}_{-} & =0, \quad \bar{e}_{-}=0, \quad \phi_{-}=-\sqrt{P^{2}} v_{(0)}=0, \\
\Theta & =\sqrt{P^{2}} u^{(0)}=0
\end{aligned}
$$

and the first class constraints in (44) are

$$
\begin{aligned}
\bar{\pi}_{+}= & 0 \\
\frac{1}{4} C_{0} \equiv & \phi_{+}-\frac{\Delta_{-}}{P^{2}} \phi_{-}=\frac{1}{4} P^{2} \\
& -\sum_{\lambda^{\prime}=1}^{3}\left(v_{\left(\lambda^{\prime}\right)}^{2}+\kappa^{2} u^{\left(\lambda^{\prime}\right) 2}\right)-\frac{\Delta_{+}}{2}+\frac{\Delta_{-}^{2}}{4 P^{2}}=0
\end{aligned}
$$

with $\Delta_{+}=\left(m_{10}^{2}+m_{20}^{2}\right)$. Notice that in $C_{0}$ we have deleted terms of square of constraints. They are the first class constraints that commute strongly with the second class constraints. This result is similar to what happens when using Dirac brackets: we can put to zero second class constraints in the expression of the first class ones. However the canonical transformation method allows us to work in the complete phase space.

The canonical quantization is obtained by imposing the commutation relations on the canonical pairs

$$
\left[\bar{e}_{i}, \bar{\pi}_{j}\right]=-i \delta_{i j}, \quad\left[\bar{x}^{\mu}, P_{\nu}\right]=-i \delta^{\mu}{ }_{\nu}, \quad\left[u^{(\lambda)}, v_{(\rho)}\right]=-i \delta_{\rho}^{\lambda},
$$

and requiring the first class constraint (50) as the physical state condition. Concerning the second class constraints, a non-Hermitian combination of Eqs. (49), following GuptaBleuler, is required so that we have

$$
\frac{1}{\sqrt{2}}\left(\bar{e}_{-}-i \bar{\pi}_{-}\right)\left|\psi_{\text {phys }}\right\rangle=0, \quad a_{(0)}\left|\psi_{\text {phys }}\right\rangle=0
$$

where we have defined the operators $a_{(\lambda)}$, for $\lambda=0,1,2,3$, as

$$
a_{(\lambda)}=\frac{1}{\sqrt{2 \kappa}}\left(v_{(\lambda)}-i \kappa u_{(\lambda)}\right)
$$

such that

$$
\left[a_{(\lambda)}, a_{(\rho)}^{\dagger}\right]=-g_{\lambda \rho} .
$$

These two conditions imply

$$
\begin{aligned}
\left\langle\psi_{\text {phys }}\left|\bar{e}_{-}\right| \psi_{\text {phys }}\right\rangle & =\left\langle\psi_{\text {phys }}\left|\bar{\pi}_{-}\right| \psi_{\text {phys }}\right\rangle=\left\langle\psi_{\text {phys }}\left|u^{(0)}\right| \psi_{\text {phys }}\right\rangle \\
& =\left\langle\psi_{\text {phys }}\left|v_{(0)}\right| \psi_{\text {phys }}\right\rangle=0
\end{aligned}
$$

and are satisfied, in the coordinate representation, by a Gaussian dependence of the wave function on the variables $\bar{e}_{-}$and $u_{(0)}$.

Finally we require the first class constraints (50) as the physical state condition,

$$
\bar{\pi}_{+}\left|\psi_{\text {phys }}\right\rangle=0 \text {, }
$$

implying that the wave function, in the coordinate representation, does not depend on $\bar{e}_{+}$and

$$
C_{0}\left|\psi_{\text {phys }}\right\rangle=0
$$

where

$$
C_{0}=P^{2}-4 \kappa \sum_{\lambda^{\prime}=1}^{3}\left(a_{\left(\lambda^{\prime}\right)}^{\dagger} a_{\left(\lambda^{\prime}\right)}+a_{\left(\lambda^{\prime}\right)} a_{\left(\lambda^{\prime}\right)}^{\dagger}\right)-2 \Delta_{+}+\frac{\Delta_{-}^{2}}{P^{2}} .
$$

Notice that for unequal masses, the propagator has the general form

$$
\frac{1}{M_{1}^{2}-M_{2}^{2}}\left[\frac{1}{p^{2}-M_{1}^{2}}-\frac{1}{p^{2}-M_{2}^{2}}\right]
$$

where $M_{1}^{2}$ and $M_{2}^{2}$ are two solutions in $P^{2}$ of the primary constraint (57). Therefore, due to the minus sign in the second term, the theory in general contains ghosts. The Hilbert space would be the direct sum of two Hilbert spaces: one with positive norm and the second one with negative norm. As soon as one adds interaction terms in the field theory Lagrangian, instabilities can occur and special care has to be taken [25].

For this reason we limit the study of the spectrum in the simplest case of equal masses $\Delta_{-}=0$ so that the wave equation becomes quadratic in the momentum. Using (57) the mass operator $\mathcal{M}^{2}$ is given by

$$
\mathcal{M}^{2}=8 \kappa\left[\sum_{\lambda^{\prime}=1}^{3} a_{\left(\lambda^{\prime}\right)}^{\dagger} a_{\left(\lambda^{\prime}\right)}+\frac{3}{2}+\frac{m_{0}^{2}}{2 \kappa}\right]
$$

Standard procedure allows us to build the quantum states of the internal space corresponding to a three-dimensional 
harmonic oscillator. For increasing occupation numbers one can build states of increasing internal angular momentum that can be given by the representation of $\mathrm{O}(3)$.

The Lorentz generators

$$
M_{\mu \nu}=x_{\mu} p_{\nu}-x_{\nu} p_{\mu}+r_{\mu} q_{\nu}-r_{\nu} q_{\mu}
$$

expressed in terms of the new canonical variables are giving the massive representation,

$$
\begin{aligned}
& M_{i j}=\bar{x}_{i} P_{j}-\bar{x}_{j} P_{i}+T_{i j}, \\
& M_{0 i}=\bar{x}_{0} P_{i}-\bar{x}_{i} P_{0}+\frac{P^{j}}{P^{0}+\sqrt{|\mathbf{P}|^{2}}} T_{j i},
\end{aligned}
$$

where $T_{\rho^{\prime} \lambda^{\prime}}$ is the $\mathrm{O}(3)$ little group generator,

$$
T_{\rho^{\prime} \lambda^{\prime}}=u_{\left(\rho^{\prime}\right)} v_{\left(\lambda^{\prime}\right)}-u_{\left(\lambda^{\prime}\right)} v_{\left(\rho^{\prime}\right)} .
$$

\section{B. Massless sector}

Let us now consider the quantization of the sectors with $P^{2}=0$. In both sectors for the space-time variables all constraints are first class and given by

$$
\begin{gathered}
\pi_{i}=0, \quad P^{2}=0, \\
q^{2}+k^{2} r^{2}-m_{0}^{2}=0, \\
(P \cdot r)=0, \quad(P \cdot q)=0
\end{gathered}
$$

or the equivalent combinations

$$
\begin{aligned}
& \pi_{ \pm}=0, \quad \phi_{+}=0, \quad \phi_{-}=-(P \cdot q)=0, \\
& \Theta=(P \cdot r)=0, \quad \chi=\frac{1}{2} P^{2}=0 .
\end{aligned}
$$

The construction of the Hilbert space can be performed by following the covariant quantization of the electromagnetic field, introducing four polarization vectors: the first $\epsilon_{(0)}{ }^{\mu}$ coincides with a timelike vector $n^{\mu}$ with $n^{2}=1$, then we consider two vectors $\epsilon_{\left(\lambda^{\prime}\right)}{ }^{\mu}, \lambda^{\prime}=1,2$ orthogonal to $P^{\mu}$ and $n^{\mu}$, and finally the fourth one, $\epsilon_{(3)^{\mu}}{ }^{\mu}=\frac{P^{\mu}-n^{\mu}(P \cdot n)}{(P \cdot n)}$, orthogonal to the previous ones. These four vectors are orthonormal

$$
\epsilon_{(\lambda)^{\mu}} \epsilon_{(\rho)}^{\nu} \eta_{\mu \nu}=\eta_{(\lambda)(\rho)} .
$$

Using them we make a canonical transformation generated by

$$
W(P, q, \bar{x}, u)=P_{\mu} \bar{x}^{\mu}+u^{(\lambda)} \epsilon_{(\lambda)}{ }^{\mu} q_{\mu} .
$$

Here we take $n^{\mu}=(1,0,0,0)$ and the polarization vectors as

$\epsilon_{(\lambda)}{ }^{\mu}=\left(\begin{array}{cccc}1 & 0 & 0 & 0 \\ 0, & 1-\frac{P_{1}{ }^{2}}{\mid \mathbf{P}\left(|\mathbf{P}|+P_{3}\right)}, & -\frac{P_{1} P_{2}}{|\mathbf{P}|\left(|\mathbf{P}|+P_{3}\right)}, & -\frac{P_{1}}{|\mathbf{P}|} \\ 0, & -\frac{P_{1} P_{2}}{|\mathbf{P}|\left(|\mathbf{P}|+P_{3}\right)}, & 1-\frac{P_{2}{ }^{2}}{|\mathbf{P}|\left(|\mathbf{P}|+P_{3}\right)}, & -\frac{P_{2}}{|\mathbf{P}|} \\ 0, & \frac{P_{1}}{|\mathbf{P}|}, & \frac{P_{2}}{|\mathbf{P}|}, & \frac{P_{3}}{|\mathbf{P}|}\end{array}\right)$.

It follows that

$x^{\mu}=\frac{\partial W}{\partial P_{\mu}}=\bar{x}^{\mu}+u^{(\lambda)} \frac{\partial \epsilon_{(\lambda)}^{\nu}}{\partial P_{\mu}} q_{\nu}, \quad \bar{P}_{\mu}=\frac{\partial W}{\partial \bar{x}^{\mu}}=P_{\mu}$,

$r^{\mu}=\frac{\partial W}{\partial q_{\mu}}=u^{(\lambda)} \epsilon_{(\lambda)^{\mu}}, \quad v_{(\lambda)}=\frac{\partial W}{\partial u^{(\lambda)}}=\epsilon_{(\lambda)^{\mu}} q_{\mu}$.

After quantization the commutation relations between the position and momentum operators are

$$
\left[\bar{x}^{\mu}, P_{\nu}\right]=-i \delta^{\mu}{ }_{\nu}, \quad\left[u^{(\lambda)}, v_{(\rho)}\right]=-i \delta_{\rho}^{\lambda}
$$

and the annihilation $a_{(\lambda)}$ and creation operators $a_{(\lambda)}^{\dagger}$ are given by

$$
a_{(\lambda)}=\frac{1}{\sqrt{2 \kappa}}\left(v_{(\lambda)}-i \kappa u_{(\lambda)}\right), \quad a_{(\lambda)}^{\dagger}=\frac{1}{\sqrt{2 \kappa}}\left(v_{(\lambda)}+i \kappa u_{(\lambda)}\right)
$$

such that

$$
\left[a_{(\lambda)}, a_{(\rho)}^{\dagger}\right]=-g_{\lambda \rho} .
$$

The constraints on the einbein sector give for sector 2

$$
\bar{\pi}_{+}\left|\psi_{\text {phys }}\right\rangle=0, \quad \bar{\pi}_{-}\left|\psi_{\text {phys }}\right\rangle=0,
$$

which imply, in coordinate representation, that the wave function does not depend on the einbein variables. Instead, for the sector 3 we have

$$
\bar{\pi}_{+}\left|\psi_{\text {phys }}\right\rangle=0, \quad \bar{a}\left|\psi_{\text {phys }}\right\rangle=\left(\bar{e}_{-}+i \bar{\pi}_{-}\right)\left|\psi_{\text {phys }}\right\rangle=0 .
$$

In this case the wave function, in the coordinate representation, does not depend on $\bar{e}_{+}$and is a Gaussian function in the variable $\bar{e}_{-}$.

The constraints (65) become physical state conditions on the quantum states

$$
P^{2}\left|\psi_{\text {phys }}\right\rangle=0, \quad\left(\sum_{\lambda^{\prime}=1}^{2} a_{\left(\lambda^{\prime}\right)}^{\dagger} a_{\left(\lambda^{\prime}\right)}-\beta\right)\left|\psi_{\text {phys }}\right\rangle=0 .
$$


Here $\beta$ is the parameter taking into account possible ambiguity in defining the quantum operator $a_{(\lambda)}^{\dagger} a_{(\lambda)}+\frac{m_{0}^{2}}{2 k}$. Note that we have included also the mass term in this ambiguity. Finally the constraints on the transversality to the total momentum (66) are imposed à la Gupta Bleuler,

$$
\left(a_{(0)}-a_{(3)}\right)\left|\psi_{\text {phys }}\right\rangle=0 \text {. }
$$

Therefore the construction of the Hilbert space can be performed by following the covariant quantization of the electromagnetic field. The general solution of Eq. (78) can be written as

$$
\left|\psi_{\text {phys }}\right\rangle=\left|\psi_{T}\right\rangle \otimes\left|\phi_{0}\right\rangle+\sum_{n \neq 0} c_{n}\left|\phi_{n}\right\rangle,
$$

where $n$ denotes the number of longitudinal $a_{(3)}$ and scalar $a_{(0)}$ oscillators and $\left|\psi_{T}\right\rangle$ is built with the transverse operators $a_{(1)}^{\dagger}$ and $a_{(2)}^{\dagger}$. All the states $\left|\varphi_{n}\right\rangle$ with $n \neq 0$ have zero norm and therefore at the end $\left|\psi_{\text {phys }}\right\rangle$ has positive definite norm.

Summing up, only $\left|\psi_{T}\right\rangle$ contributes to the observables and the physical states exist for non-negative integers of $\beta$. If we choose an integer $\beta=n \geq 0$, the states have helicities ranging

$$
n, n-2, \ldots,-n+2,-n
$$

For instance, the states for $\beta=1$ are

$$
\frac{1}{\sqrt{2}}\left(a_{(1)}^{\dagger} \pm i a_{(2)}^{\dagger}\right)|p ; 0\rangle
$$

where

$$
|p ; 0\rangle=|p\rangle \otimes|0\rangle
$$

with $P_{\mu}|p\rangle=p_{\mu}|p\rangle$. They are corresponding to helicity \pm 1 states as can be checked from the form of the helicity operator,

$$
\Lambda=-i\left(a_{(1)}^{\dagger} a_{(2)}-a_{(2)}^{\dagger} a_{(1)}\right)
$$

Indeed the Lorentz generators

$$
M_{\mu \nu}=x_{\mu} p_{\nu}-x_{\nu} p_{\mu}+r_{\mu} q_{\nu}-r_{\nu} q_{\mu}
$$

once use is made of Eq. (71), become the massless representation as

$$
\begin{aligned}
& M_{12}=\bar{x}_{1} P_{2}-\bar{x}_{2} P_{1}+\Lambda, \\
& M_{23}=\bar{x}_{2} P_{3}-\bar{x}_{3} P_{2}+\frac{P_{1}}{P_{3}+|\mathbf{P}|} \Lambda, \\
& M_{31}=\bar{x}_{3} P_{1}-\bar{x}_{1} P_{3}+\frac{P_{2}}{P_{3}+|\mathbf{P}|} \Lambda, \\
& M_{01}=\bar{x}_{0} P_{1}-\bar{x}_{1} P_{0}-\frac{P_{0} P_{2}}{|\mathbf{P}|\left(P_{3}+|\mathbf{P}|\right)} \Lambda, \\
& M_{02}=\bar{x}_{0} P_{2}-\bar{x}_{2} P_{0}+\frac{P_{0} P_{1}}{|\mathbf{P}|\left(P_{3}+|\mathbf{P}|\right)} \Lambda, \\
& M_{03}=\bar{x}_{0} P_{3}-\bar{x}_{3} P_{0},
\end{aligned}
$$

where $P_{0}= \pm|\mathbf{P}|$ corresponding to its signs.

If we identify $\beta=-2-\frac{m_{0}^{2}}{k}$, only $m_{0}^{2}<0$ can allow massless solutions for the physical states. The $P^{2}=0$ sector at the quantum level, and also at the classical level, is empty for positive rest mass $m_{0}^{2}>0$. Nontrivial massless sectors appear by choosing the tachyonic rest mass parameter $m_{0}^{2}<0$.

\section{CONCLUSIONS AND OUTLOOK}

In this paper we have studied a model of two interacting relativistic particles via a harmonic potential at classical and quantum levels. The model contains three parameters, the two rest masses of the particles and the frequency of the harmonic oscillator. When the rest masses are different, the model has only the massive sector. At quantum level the spectrum in general contains a branch with ghosts. Instead if the rest masses are equal, the model has the massive and massless sectors. At quantum level the massive sector has a spectrum of increasing masses with higher internal spin. The massless sector is nontrivial at classical and quantum level when we consider tachyonic rest masses. We have also given the two Noether gauge transformations in the massless sector of equal rest masses. One of the transformations is the worldline diffeomorphism. For the second gauge transformation, that has an open algebra, we have not yet found a clear geometrical interpretation.

It is interesting to ask what happens when the distance among the particles is constrained to be lightlike. This situation does not occur for the harmonic potential as we have seen. Lightlike sectors with $r^{2}=0$, in addition to the massive sector, appear, for example, if we consider a quartic potential $V\left(r^{2}\right)=k\left(r^{2}\right)^{2}$. Being more precise there are four sectors with the lightlike constraint; among these there is one with the following set of constraints:

$$
P^{2}=0, \quad q^{2}=0, \quad r^{2}=0, \quad(P \cdot r)=0, \quad(P \cdot q)=0 .
$$

The same constraints appear in the rigid string model [26-28]. Therefore, our model, for a suitable choice of the 
potential, has a sector that describes the rigid motion of a string.

Our model can be thought of as a sort of effective theory describing possible interaction among two particles. It does not give any prescription to determine the potential. The form of the potential could be chosen from phenomenological reasons or in the best scenario could come from a fundamental theory describing the higher energy degrees of freedom.

We also think that the analysis of this model, presented here, could be a useful example to examine other dynamical systems with sectors, like bigravity theories.

In a work in progress we will consider how we can construct the gauge transformations from the constraint structure. We will also consider the gauge structure of the model by considering the solutions of the classical master equation.

\section{ACKNOWLEDGMENTS}

We acknowledge Jorge Alfaro, Luis Alvarez-Gaume, Max Banados, Carles Batlle, Eric Bergshoeff, Roberto Casalbuoni, Marc Henneaux, Luca Lusanna, Paul Townsend, and Jorge Zanelli for comments and discussions. J. G. acknowledges the hospitality at the Universidad Católica de Santiago de Chile where this work has started and CERN for hospitality and partial financial support. J. G. also acknowledges financial support from FPA 2010-20807, 2009 SGR502, CPAN, Consolider CSD 2007-0042, and Eplanet for partial financial support for the stay in Chile.
[1] M. Henneaux and C. Teitelboim, Quantization of Gauge Systems (University Press, Princeton, NJ, 1992).

[2] M. Henneaux, C. Teitelboim, and J. Zanelli, Phys. Rev. A 36, 4417 (1987).

[3] A. Shapere and F. Wilczek, Phys. Rev. Lett. 109, 160402 (2012).

[4] F. Wilczek, Phys. Rev. Lett. 109, 160401 (2012).

[5] T. L. Curtright and C. K. Zachos, arXiv:1311.6147.

[6] E. A. Bergshoeff, S. de Haan, O. Hohm, W. Merbis, and P. K. Townsend, Phys. Rev. Lett. 111, 111102 (2013).

[7] M. Bañados, C. Deffayet, and M. Pino, Phys. Rev. D 88, 124016 (2013).

[8] S. Alexandrov, Gen. Relativ. Gravit. 46, 1639 (2013).

[9] L. Lusanna, Riv. Nuovo Cimento 14, 1 (1991).

[10] M. Banados, L. J. Garay, and M. Henneaux, Phys. Rev. D 53, R593 (1996); M. Banados, L. J. Garay, and M. Henneaux, Nucl. Phys. B476, 611 (1996).

[11] J. Saavedra, R. Troncoso, and J. Zanelli, J. Math. Phys. (N.Y.) 42, 4383 (2001); F. de Micheli and J. Zanelli, J. Math. Phys. (N.Y.) 53, 102112 (2012).

[12] E. Ivanov, L. Mezincescu and P. K. Townsend, J. High Energy Phys. 01 (2006) 143.

[13] K. Kamimura and T. Shimizu, Prog. Theor. Phys. 58, 383 (1977).

[14] K. Kamimura, Prog. Theor. Phys. 58, 1947 (1977).
[15] D. Dominici, J. Gomis, and G. Longhi, Nuovo Cimento Soc. Ital. Fis. B 48, 152 (1978).

[16] D. Dominici, J. Gomis, and G. Longhi, Nuovo Cimento A 48, 257 (1978).

[17] S. Shanmugadhasan, J. Math. Phys. (N.Y.) 14, 677 (1973).

[18] L. Castellani, D. Dominici, and G. Longhi, Nuovo Cimento A 48, 91 (1978).

[19] C. Batlle and N. Roman-Roy, Anales de Fisica Serie A, Fenomenos e interacciones (1981-1991): publicacion de la Real Sociedad Espanola de Fisica 84, 131 (1988).

[20] Y. S. Kalashnikova and A. V. Nefediev, Yad. Fiz. 60, 1529 (1997) [, Phys. At. Nucl. 60, 1389 (1997)].

[21] I. A. Batalin and G. A. Vilkovisky, Phys. Lett. 102B, 27 (1981).

[22] M. Henneaux, Nucl. Phys. B, Proc. Suppl. 18, 47 (1990).

[23] J. Gomis, J. Paris, and S. Samuel, Phys. Rep. 259, 1 (1995).

[24] J. Gomis, K. Kamimura (work in progress).

[25] S. W. Hawking and T. Hertog, Phys. Rev. D 65, 103515 (2002).

[26] R. Casalbuoni and G. Longhi, Nuovo Cimento A 25, 482 (1975).

[27] R. Casalbuoni, D. Dominici, and G. Longhi, Nuovo Cimento A 32, 265 (1976).

[28] K. Kamimura and D. Shiseki, Nucl. Phys. B806, 489 (2009). 